УДК 331.3

DOI $10.21661 / \mathrm{r}-472971$

\title{
М.В. Михайлюк
}

\section{ФАКТОРЫ И УСЛОВИЯ РАЗВИТИЯ ВНУТРЕННЕ ОРИЕНТИРОВАННЫХ И ЭКСПОРТНЫХ ЦЕПЕЙ ПОСТАВОК В ИНТЕРНЕТ-ТОРГОВЛЕ РФ}

\begin{abstract}
Аннотация: в статье автором обосновывается, что интенсивное развитие рынка, повыщение конкурентоспособности внутреннего производства, более интенсивный переход производителей из офлайна в онлайн сформируют мощзнейшие предпосылки для развития рынка и обеспечивающей его логистической инфраструктуры, прежде всего, региональной.
\end{abstract}

Ключевые слова: внутренне ориентированная цеепь поставок, экспортная цеепь поставок, интернет-торговля, производство.

\section{V. Mikhailiuk}

\section{FACTORS AND CONDITIONS OF DEVELOPMENT}

\section{OF INTERNALLY ORIENTED AND EXPORT SUPPLY CHAINS IN THE ONLINE TRADE OF THE RUSSIAN FEDERATION}

Abstract: in the article the author proves that intensive market development, increasing the competitiveness of domestic production, more intensive transition of producers from offline to online will form the most powerful prerequisites for the development of the market and its logistics infrastructure, primarily regional.

Keywords: internally oriented supply chain, export supply chain, online trade, production.

Основная проблема конкуренции российских интернет-магазинов и зарубежных на внутреннем рынке состоит в том, что мы имеем «рынок перепродавцов. «По факту, российский е-соmmerce торгуют тем же, что и зарубежные интернет-магазины - только прибавляя к цене зарубежного товара, и без того уже 
прошедшего цепочку посредников, свою собственную торговую наценку» [6, с. 4].

Отсюда, российский онлайн-ритейл работает по схеме более длинной цепи поставок и не может использовать преимущества локализации и выстроить цепи с минимальным числом звеньев и коротким плечом доставки. Решение этой проблемы требует развития в России массового широкомасштабного производства продукции, конкурентоспособной как по цене, так и по качеству, сбыт которой позволит покрыть потребительский спрос во внутреннем e-commerce и потеснить зарубежный онлайн-ритейл. Этот процесс может и должен быть простимулирован ужесточением норм для кросс-бордера, отсутствие которого сегодня ставит в неравное положение российские и зарубежные интернет-магазины.

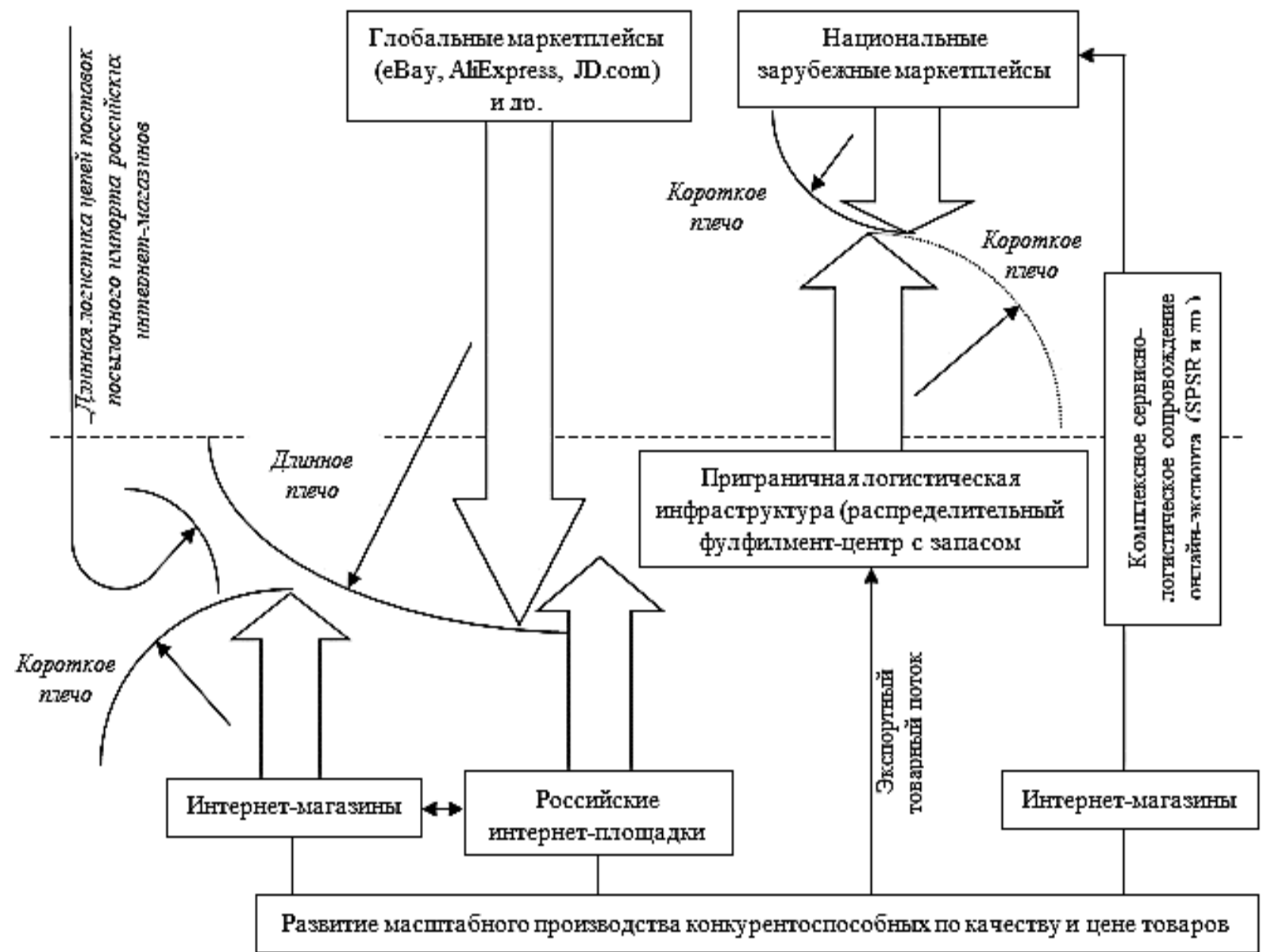

Рис. 1. Факторы и условия развития внутренне-ориентированных и экспортных цепей поставок в интернет-торговле РФ 
На схеме рисунка 1 представлены условия и факторы развития экспортных и внутренне-ориентированных цепей поставок в условиях продолжающейся экспансии в Россию трансграничного онлайн-ритейла, а также ограниченных возможностей выхода российских производителей на внешние рынки.

Жесткое конкурентное давление со стороны зарубежных интернет-площадок не позволяет отечественному онлайн-ритейлу должным образом масштабироваться, что увеличивает стоимость операционной обработки заказов и при неравной фискальной нагрузке сужает возможности для рентабельной ценовой конкуренции.

Таким образом, рост российского е-соmmerce требует более глубокой локализации и фокусировки на конкурентоспособном производстве отдельных товарных категорий, что обеспечит устойчивое ценовое и ассортиментное доминирование на внутреннем рынке, обеспечивая конкурентное преимущество перед зарубежным онлайн-ритейлом.

В немалой степени это обеспечит расширение возможностей рентабельного функционирования отечественных маркетплейсов, которые отходят от чистой транзакционной модели, инвестируют в сервисы, логистику и самостоятельную розницу (заборная логистика), развитие которой требует крайне избирательного формирования ассортимента, который вкупе с сервисами позволяет повысить процент возвращающегося трафика.

То есть, имеет место достаточно тесное переплетение множества факторов и условий, формирующих потенциал конкурентного роста российского e-commerce и построения внутренне-ориентированных цепей поставок, позволяющих максимально использовать все логистические преимущества локализации. В противном случае, Россия будет терять внутренний рынок интернет-торговли, на котором помимо китайских поставщиков, примерно такой же потенциал для агрессивного входа и развития имеют турецкий производители и бренды, «которые также смогут расширить свое присутствие на российском рынке в случае падения спроса на американские и европейские товары» [4, с. 65]. 
В современный период значительный потенциал расширения внутренней интернет-торговли может дать выход в регионы, которые сегодня не имеют достаточного покрытия логистической инфраструктурой. При низком проценте предоплаты в регионах многие зарубежные поставщики (с высоким средним чеком) не рискуют отправлять товар, оборачиваемость которого автоматически возрастает вдвое. Это требует дополнительного финансирования оборотного капитала, к которому обращаются некоторые интернет-площадки (например, Wikimart), которые сегодня самостоятельно развивают розничное направление и сами выступают продавцами, имея сильную экспертизу в этом вопросе.

Все это позволяет заключить, что интенсивное развитие рынка, повышение конкурентоспособности внутреннего производства, более интенсивный переход производителей из офлайна в онлайн сформируют мощнейшие предпосылки для развития рынка и обеспечивающей его логистической инфраструктуры, прежде всего, региональной.

Аналогичная ситуация складывается в сфере российского онлайн-экспорта, который достаточно перспективен, например для приграничных райнов, которыми, например, для Санкт-Петербурга являются Скандинавские страны и Прибалтика. Формирование в приграничной зоне большого фулфилмент-центра, аккумулирующего значительные товарные запасы (Сочи, Новороссийск) позволит существенно увеличить объем экспорта, используя, в том числе, преимущества короткого транспортного плеча [7, с. 23].

В целом, основным - базовым катализатором роста онлайн-экспорта сегодня является повышение конкурентоспособности внутреннего производства, ориентированного на потребительский сектор, а также расширение сервисного сопровождения при выходе на внешние рынки, например через зарубежные национальные торговые площадки.

Реализация таких планов через глобальные площадки, например AliExpress сегодня продвигается крайне тяжело по причине отсутствия готового юридического решения данного вопроса, а также понятной схемы, «которая позволит 
легальным российским компаниям в рублевой зоне продавать через AliExpress за границу и получать при этом валюту» [5, с. 65].

Тем не менее, крупные российские логистические операторы (например, SPSR Express) сегодня наращивают активность в части оказания расширенного комплекса услуг для российских экспортно-ориентированных производственных компаний в выводе их на онлайн-площадки, включая «содействие в создании собственных интернет-магазинов и привлечении трафика на их страницы, ведение офлайновых проектов, например открытие шоу-румов» и др. [2, с. 45].

Это позволит поставщикам максимально сконцентрироваться на производстве конкурентоспособной продукции, задачи экспорта, размещения и доставки которой будут полностью решаться логистическим оператором. На начало 2017 г., SPSR Express была разработана дорожная карта реализации таких проектов, определен пул потенциально привлекательных для логистического оператора производственных компаний. В современный период, уже имеются договоренности с торговой площадкой «JD Worldwide, заключен договор с Amazon, на текущий момент ведутся переговоры с DHgate и eBay, в перспективе будет налаживаться сотрудничество с французскими Cdiscount и Pixmania, а также американскими Newegg и Jet и т. д.» [2, с. 44].

Таким образом, развитие экспортно-ориентированных цепей поставок сегодня опосредованно целым комплексом процессов, в ходе которых продуктовая специализация и работа с ассортиментом производителей сопровождается не менее глубокими изменениями функционала логистических операторов.

Последние берут на себя более широкий комплекс услуг «по поддержке продаж, логистике, помощь в создании листинга товара, его публикации, разработке продуктовых каталогов для различных площадок» [2, с. 44]. Однако, на наш взгляд, более важным фактором развития цепей поставок и интенсификации экспортных товарных потоков выступает именно широкомасштабное развитие конкурентоспособных производств, которое потянет за собой преобразование сервисного и логистического сопровождения экспорта, особенно в условиях 
объединения этого разрозненного функционала самими логистическими операторами.

В настоящее время, как показали результаты анализа исследовательской компании Data Insight и eBay, российский онлайн экспорт представлен преимущественно предприятиями малого и среднего бизнеса с узким спектром достаточно стандартных категорий (fashion, electronic и collectables (коллекционные товары)), четверть объема которого продается через маркетплейсы, прежде всего, еВау [12]. Сегодня площадка еВау существенно расширила инфраструктуру сервиса и может «быстро наращивать» объем продаж, а также активно взаимодействует с Почтой России, DHL и другими компаниями, что позволяет ей создавать адекватный логистический экспортный продукт.

Потенциал развития экспорта в условиях ограниченности емкости национального рынка является «безграничным» при условии, что национальному производителю есть с чем на него выйти. Развитие логистики, логистической инфраструктуры, цепей поставок в потребительском сегменте рынка является вторичным по отношению к решению этой на порядок более сложной проблемы. Именно формирование условий для развития конкурентоспособного реально производящего сектора экономики формирует условия, при которых наращивание инвестиций в логистику, институциональное развитие логистических сервисов срочной доставки происходит в условиях, когда внутри экономики происходит рост товарной массы, востребованной покупателем, под которую формируется и развивается логистика. Десинхронизация данных процессов в перспективе формирует оси структурного неравновесия в экономике, а также может приводит к упразднению моделей роста логистики, например спекулятивной модели роста складского девелопмента, которая до кризиса конца 2014 г. и последовавшего за ним сжатия спроса была ориентирована на отчасти искусственный рост конечного потребления, кредитная прививка которого (система потребительского кредитования, дешевое фондирование корпоративного сегмента) лишь усиливала иллюзию социального и экономического благополучия. Достаточно подробно на 
уровне логистики данная проблема рассмотрена, в частности, в исследованиях А.У. Альбекова и С.Н. Резникова $[1 ; 9-11]$.

В контексте рассматриваемых нами проблем современной трансформации многоканальной торговли связь между конкурентоспособностью внутреннего производства, институциональным прогрессивным преобразованием онлайн-ритейла (не просто появление маркетплейсов, а уже эволюционное изменение их модели) и развитием логистики становится еще теснее именно в рамках текущей фазы рыночного цикла. Именно сегодня жесткая конкуренция в цифровых каналах сбыта, давление со стороны глобальных онлайн-площадок заставляют маркетплейсы трансформировать свою транзакционную бизнес-модель - предложить покупателю больше сервиса, логистики, расширение ассортимента за счет маркетплейса. Источником такого расширения, особенно для национальных торговых площадок может выступить конкурентоспособное интересное по ассортименту предложение внутреннего производителя. В противном случае, «добор» ассортимента, как это происходит сегодня будет происходить на глобальных рынках. Развитие по этому сценарию, так или иначе, будет сужать синергию этого процесса, которая может формироваться по схеме: малое число звеньев + короткая и быстрая логистика + ускорение сокращение производственного цикла + низкая цена.

В этом случае, экономика получит дополнительную точку роста, а из публичного поля научно-практического дискурса будет более спешно вымываться фразеология, подчеркивающая необходимость оплаты российским покупателем неэффективности ошибочных бизнес-моделей отечественных интернет-магазинов [3]. Сегодня такая апология объективной рыночной силы зарубежного онлайн-ритейла на российском рынке в определенном и немалом смысле имеет право на существование и имеет место быть.

\section{Список литературы}

1. Альбеков А.У. Глобализация vs регионализация современных цепей поставок: Моногр. / А.У. Альбеков, С.Н. Резников. - Ростов н/Д: Издательско-полиграфический комплекс РГЭУ (РИНХ), 2014. 
2. Борисов A. SPSR Express: мы не видим непреодолимых барьеров для развития онлайн-экспорта // Retail\&Loyalty. - 2017. - №3 (66). - C. 45.

3. Димитров И. Российские онлайн-маркетплейсы: как им доминировать на международном рынке? // Retail\&Loyalty. - 2017. - №7 (70). - C. $42-45$.

4. Завадский M. AliExpress: кризис как катализатор развития онлайн-ритейла в России // Retail\&Loyalty. - 2015. - №2 (47). - С.65.

5. Завадский M. AliExpress: переход от «модели Таobao» к ранней версии «Tmall» в России завершен // Retail\&Loyalty. - 2017. - №1 (64). - C.65.

6. Иванов А. Российский рынок е-commerce 2016: ожидания и реальные перспективы // Retail\&Loyalty. - 2016. - №3 (57). - C. 4.

7. Костыгин Д. Юлмарт строит соцсеть на мессинджиговой платформе в формате B2C // Retail\&Loyalty. - 2017. - №4 (67). - C.23.

8. Резников С.Н. Глобальная перестройка цепей поставок в системе международной товарно-сбытовой кооперации / С.Н. Резников // Проблемы, тенденции и перспективы развития современной логистической науки: Коллективная монография / Под общ. Ред. Н.А. Адамова. - М.: Институт исследования товародвижения и конъюнктуры оптового рынка, 2013.

9. Резников С.Н. Мировая торговля и кризис перепроизводства в системе глобального товарного обмена: Моногр. / С.Н. Резников; под науч. ред. А.У. Альбекова. - Ростов н/Д: Издательско-полиграфический комплекс РГЭУ (РИНХ), 2014.

10. Резников С.Н. Современная трансформация глобальных цепей поставок: настоящее и будущее: Моногр. / С.Н. Резников; под науч. ред. А.У. Альбекова. - Ростов н/Д: Издательско-полиграфический комплекс РГЭУ (РИНХ), 2014.

11. Резников С.Н. Стимулирование спроса в экономике как фактор повышения устойчивости современных производственно-сбытовых цепей / С.Н. Резников // Современные логистические технологии и стратегии (торговый комплекс): Моногр. / Науч. ред. А.У. Альбеков. - Ростов н/Д: Издательско-полиграфический комплекс РГЭУ (РИНХ), 2013. 
12. Экспорт товаров через интернет: Data Insight и eBay // По данным портала E-pepper.ru - нишевый информационный онлайн-ресурс, посвященный электронной коммерции в России [Электронный ресурс]. - Режим доступа: https://e-pepper.ru/news/eksport-tovarov-cherez-internet-issledovanie data-insight-iebay.html (дата обращения: 14.01.2018).

\section{References}

1. Albekov A.U. Globalization vs regionalization of modern supply chains: Monograph // A.U. Albekov, S.N. Reznikov. - Rostov n/D: Publishing and printing complex at Rostov state economic University (RINH), 2014.

2. Borisov A. SPSR Express: we do not see any insuperable barriers to the development of an online import / export/ Retail\&Loyalty. - 2017. - №3 (66). - P. 45.

3. Dimitrov I. Russian online marketplaces: how can they dominate the international market? // Retail \& Loyalty. - 2017. - №7 (70). - P. 42-45.

4. Zavadsky M. AliExpress: crisis as a catalyst for the development of online retail in Russia // Retail\&Loyalty. - 2015. - №2 (47). - P. 65.

5. Zavadsky M. Aliexpress: the transition from» Taobao model «to the early version of» Tmall " in Russia is completed // Retail\&Loyalty. - 2017. - №1 (64). - P. 65.

6. Ivanov A. Russian market e-commerce 2016: expectations and real prospects // Retail\&Loyalty. - 2016. - №3 (57). - P. 4.

7. Costigan D. Hardware builds on messengerboy social network platform B2C format // Retail\&Loyalty. - 2017. - №4 (67). - P. 23.

8. Reznikov S.N. Global restructuring of supply chains in the system of international commodity-marketing cooperation / S.N. Reznikov // Problems, trends and prospects of development of modern logistics science: collective monograph / Under Ls. N.A. Adamova. - M.: Institute of research of goods movement and wholesale market, 2013.

9. Reznikov S.N. World trade and the crisis of overproduction in the system of global commodity exchange: Monograph / S.N. Reznikov; under the science. red. A.U. Albekova. - Rostov n/D: Publishing and printing complex at Rostov state economic University (RINH), 2014. 
10. Reznikov S.N. Modern transformation of global supply chains: present and future: Monograph / S.N. Reznikov; under the science. red. A.U. Albekova. - Rostov $\mathrm{n} / \mathrm{D}$ : Publishing and printing complex at Rostov state economic University (RINH), 2014.

11. Reznikov S.N. Stimulation of demand in the economy as a factor of increasing the stability of modern production and supply chains / S.N. Reznikov // Modern logistics technologies and strategies (shopping complex): Monogr. / Red. A.U. Albekov. - Rostov n/D: Publishing and printing complex at Rostov state economic University (RINH), 2013.

12. Export of goods via the Internet: Data Insight and eBay / / according to the portal E-pepper.ru -niche online information resource dedicated to e-Commerce in Russia [Electronic resource]. - Access mode: https://e-pepper.ru/news/eksport-tovarov-cherez-internet-issledovanie data-insight-I-ebay.html (accessed: 14.01.2018).

Михайлюк Михаил Владимирович - канд. экон. наук, доцент ФГБОУ ВО «Ростовский государственный университет путей сообщения», Россия, Ростовна-Дону.

Mikhailiuk Mikhail Vladimirovich - candidate of economic sciences, associate professor at the Rostov State Transport University, Russia, Rostov on Don. 